\title{
Educação continuada em gerontologia para agentes comunitários de saúde
}

\author{
Continuing Education in gerontology for community health agent \\ Educación continua en gerontología para los agentes comunitarios de salud
}

Nádia Placideli. Universidade Estadual Paulista Júlio de Mesquita Filho (UNESP). Botucatu, SP, Brasil. nadiaplacideli@gmail.com

(Autora correspondente)

Tania Ruiz. Universidade Estadual Paulista Júlio de Mesquita Filho (UNESP). Botucatu, SP, Brasil. truiz@fmb.unesp.br

\section{Resumo}

Objetivo: avaliar o efeito de um Programa de Educação em Gerontologia desenvolvido à Agentes Comunitários de Saúde. Métodos: estudo de intervenção (comparação antes e depois) com participação de 111 agentes comunitários, com perda de 25 (22,5\%), atuantes nas 12 unidades de saúde da família de um município do interior paulista, em 2012. Os dados foram coletados por meio de questionário sociodemográfico, instrumento de avaliação do contato dos agentes comunitários com idosos e questionário para avaliação de conhecimentos gerontológicos (Questionário Palmore-NeriCachioni). Foi desenvolvido um Programa de Educação em Gerontologia junto aos agentes comunitários, no período de 5 dias: encontros com duração de 90 minutos cada um, abordando temas relacionados à velhice e envelhecimento. Para análise dos dados, foi utilizado o programa SAS versão 9.2 para Windows. Resultados: prevaleceram entre os agentes os adultos jovens, do sexo feminino, casados, com ensino médio completo e inseridos na atividade há mais de dois anos e meio. A maioria dos agentes expôs sempre ter contato com idosos, cultivado em âmbito do trabalho e intrafamiliar e relatou experiência com a população dessa faixa etária, mais da metade referiu ter participado de educação gerontológica. O maior número de acertos ao questionário de avaliação sobre conhecimentos gerontológicos, aplicado antes, imediatamente após e depois de três meses ao desenvolvimento do Programa de Educação em Gerontologia, demonstrou melhor desempenho dos agentes na segunda e terceira aplicações desse instrumento realizadas após o programa, tornando evidente a importância de educação em gerontologia para esse público. Conclusão: promover educação em gerontologia para agentes comunitários de saúde é fundamental, pois providos de conhecimentos podem reforçar seu papel mediante a equipe de saúde e população, dentre ela, os idosos.

\begin{abstract}
Objective: to evaluate the effect of an education program in gerontology for community health agents. Methods: this intervention study (before and after comparison) included 111 community agents, lossy 25 (22.5\%), from 12 family health centers from city in the state of São Paulo in 2012. Data were collected through a sociodemographic questionnaire, to determine the formal and informal contact of the elderly group with the community health agents, as well as through a questionnaire that evaluated gerontological knowledge (Questionnaire Palmore-Neri-Cachioni). Moreover, an education program in gerontology was conducted for community agents, including 90-minute sessions over a 5-day period, which focused on topics related to old age and aging. Data analysis was performed using the SAS version 9.2 for Windows. Results: young adults, female sex, married status, high school education, and activity for more than two and a half years were common characteristics associated with community agents. Most of the agents were in contact with the elderly, to cultivate of work and within the family, and reported to have experience in dealing with this age group. Moreover, more than half of the community agents reported to have participated in gerontological education. The questionnaire assessing the knowledge on gerontology, which was applied before, immediately after, and three months after the education program in gerontology indicated better responses among the community health agents immediately after and three months after the program, thus suggesting that education in gerontology is important among these individuals. Conclusion: promoting education in gerontology among community health agents is essential, as the knowledge provided can strengthen the role of health care staff and the population in caring for the elderly.
\end{abstract}

Palavras-chave:

Sistemas de Saúde

Educação em Saúde

Envelhecimento

Gerontologia

\section{Keywords:}

Health Systems

Health Education

Aging

Gerontology
Como citar: Placideli N, Ruiz T. Educação continuada em gerontologia para agentes comunitários de saúde. Rev Bras Med Fam Comunidade. 2015;10(36)1-10.

http://dx.doi.org/10.5712/rbmfc10(36)948

\section{Fonte de financiamento:}

CAPES.

Parecer CEP: aprovado.

Conflito de interesses: declaram não haver.

Procedência e revisão por pares: revisado por pares. Recebido em: 30/05/2014. Aprovado em: 04/08/2015. 


\section{Resumen}

Objetivo: evaluar el efecto de un Programa de Educación en Gerontología desarrollado para Agentes Comunitarios de Salud. Métodos: un estudio de intervención (en comparación antes y después) con 111 agentes comunitarios, con una pérdida de 25 (22,5\%), que trabajan en 12 unidades de salud de la familia en ciudad en el estado de São Paulo en el 2012. Los datos fueron recogidos a través de cuestionarios: sociodemográficos, contacto con los profesionales con los mayores y la evaluación del conocimiento en gerontología (Palmore-Neri-Cachioni). Programa de Educación en Gerontología desarrollado abarca temas relacionados con el envejecimiento. Para el análisis de los datos se utilizó SAS versión 9.2. Resultados: prevalecieron entre estos profesionales, adultos, femenino, casado, completado la escuela secundaria. Mayoría siempre tienen contacto con los mayores y reportaron haber participado de educación gerontológica. El mayor número de respuestas correctas al cuestionario sobre conocimiento gerontológico, aplicado antes, inmediatamente después y tres meses después de que el desarrollo del Programa de Educación en Gerontología, mostró mejor rendimiento en la segunda y tercera solicitudes después del programa. Conclusión: educación en gerontológica para agentes comunitarios es fundamental, conforme a lo dispuesto conocimiento puede fortalecer su papel por parte del personal de salud y población, entre sus mayores.
Palabras clave:

Sistemas de Salud

Educación para la salud

Envejecimiento

Gerontología

\section{Introdução}

No término do século $X X$ o Brasil teve um acentuado envelhecimento da estrutura etária de sua população. À medida que o ciclo vital se alonga, aumenta a probabilidade de ocorrência das doenças crônicas não transmissíveis (DCNT) e seus comprometimentos: a grande incidência e prevalência de eventos mórbidos nos idosos resultam em demanda relativamente maior aos serviços de saúde. ${ }^{1}$ Considerando o envelhecimento populacional brasileiro, o desafio posto ao Sistema Único de Saúde (SUS) é atender à população de idosos que já apresenta uma ou mais das DCNT e se preocupar em promover assistência preventiva à população que se encontra em fases antecedentes à velhice. ${ }^{2}$

Na década de 1990 foi oficialmente criada a Estratégia Saúde Família (ESF) visando à descentralização do SUS, com a atenção primária desenvolvida por uma equipe multiprofissional composta por um médico e enfermeiro generalistas, um ou dois técnicos ou auxiliares de enfermagem e quatro a seis Agentes Comunitários de Saúde (ACS). ${ }^{3}$ Os ACS desempenham diversas ações em saúde, como cadastramento, mapeamento, identificação de microáreas de risco, entre outras, direcionadas aos diversos segmentos etários da população e, entre elas, há aquelas específicas para a população de idosos. ${ }^{4}$

Desta maneira, em estudos realizados por Bezerra et al. e Ferreira \& Ruiz, ambos constataram a ausência de conhecimentos gerontológicos pelos agentes comunitários e, a aproximação destes com educações gerontológicas esteve relacionada a melhores desempenhos em questionários aplicados ${ }^{5,6} \mathrm{O}$ aumento da proporção de idosos implica em demandas recorrentes aos serviços de saúde; dentre elas, destaca-se o provimento de educação continuada e permanente em gerontologia aos profissionais de saúde, em especial aos ACS. Compreende-se por educação continuada a transmissão de conhecimentos e a valorização da ciência como fonte para tal, com enfoque nas categorias profissionais e ênfase em promoção de cursos e treinamentos. Já a educação permanente é fundamentada na concepção de educação como transformação e aprendizagem significativa, centrada na valorização do processo de trabalho, da articulação como fonte de aquisição de conhecimento. ${ }^{7}$

É imprescindível a contribuição do ACS no atendimento aos idosos. De acordo com Vecchia ${ }^{3}$, esse profissional pode tornar-se um catalisador de recursos escondidos na comunidade para ampliar o apoio social, com papel importante na constituição de rede de cuidados no território, aumentando possibilidades de troca de afetos e ajuda pelos membros de uma mesma localidade. Nesse âmbito, os ACS desempenham papel crucial junto à população de idosos, sendo o elo entre unidade de saúde, idosos e demais profissionais que compõem a equipe ESF em busca da promoção da qualidade de vida e bem-estar desses indivíduos. 
Esta pesquisa teve como objetivo avaliar o efeito de um Programa de Educação em Gerontologia desenvolvido para Agentes Comunitários de Saúde.

\section{Métodos}

Estudo de intervenção com comparação antes e depois, realizado em Lençóis Paulista, SP, município situado no centro-oeste paulista, com população de 61.428 habitantes segundo dados do Instituto Brasileiro de Geografia e Estatística (2010). ${ }^{8}$ O município possui cobertura de 100\% (ESF). A população de estudo totalizava 111 ACS no período da coleta dos dados, atuantes nas 12 unidades de saúde da família, sendo 7 unidades ESF e 5 unidades Estratégia Agentes Comunitários de Saúde (EACS).

Os critérios de inclusão da pesquisa estiveram pautados no consentimento em participar do estudo mediante assinatura do Termo de Consentimento Livre e Esclarecido, participação em pelo menos $80 \%$ das atividades programadas. Dentre os profissionais, 22,5\% não aderiram à pesquisa, principalmente devido a licenças médicas, férias, e por não atenderem a um dos itens do critério de inclusão a pesquisa. Não houve associação estatística entre esses motivos com as diversas variáveis estudadas, como idade, nível de escolaridade, frequência de contato com idosos, entre outras.

Foram utilizados três instrumentos para coleta dos dados. O questionário sociodemográfico objetivou descrever o perfil sociodemográfico dos ACS (idade; sexo; estado civil; nível de escolaridade; anos de trabalho como ACS; renda familiar em salários mínimos) e as questões "participou de algum programa de educação gerontológica envolvendo o tema envelhecimento e velhice?"; "Se sim, você recorda de algum conteúdo aprendido?"

O instrumento Avaliação do contato dos ACS com idosos visou investigar a relação dos agentes com idosos, composto por dez questões - você possui familiares idosos? Você mora/morou com idosos? Com que frequência você tem contato com idosos? Esse contato você caracteriza como sendo (formal, cultivada apenas no trabalho; informal, cultivada apenas na família ou misto, cultivada no trabalho e na família)? Você tem alguma experiência de trabalho com idosos? Se sim, como essa experiência foi adquirida? Qual sua opinião sobre os idosos que você tem contato por meio do trabalho, quanto aos aspectos biológicos, psicológicos e sociais? Quanto você acha que o ACS contribui para que os idosos possam ter um bem-estar e uma qualidade de vida cada vez melhor? Por quê? (Descreva o motivo da resposta assinalada na questão anterior). O que você espera de um programa de educação gerontológica? Ressalta-se que esse instrumento foi criado para auxiliar na pesquisa, com base em questionário semelhante, utilizado em estudo realizado por Ferreira \& Ruiz. ${ }^{6}$

Foi empregado o instrumento para avaliação de conhecimentos gerontológicos o questionário Palmore-Neri-Cachioni, validado em 2002 em amostra com 102 professores universitários em estudo intitulado "Quem educa os idosos". O questionário é uma versão traduzida e adaptada do Palmore Aging Quis (1996), com 25 perguntas de múltipla escolha que abordam temas sobre envelhecimento e velhice, em que o maior número de acertos reflete mais conhecimento nessa área. As questões abrangem os domínios físico (totaliza seis questões), cognitivo (totaliza duas questões), psicológico (totaliza seis questões), social (totaliza sete questões), ainda há as composições, físico/psicológico (totaliza duas questões), físico/psicológico/social e psicológico/social (totalizando uma questão cada). ${ }^{9}$ 
Foi realizada educação continuada em gerontologia, para todos os sujeitos envolvidos na pesquisa, com desenvolvimento de um Programa de Educação em Gerontologia (PEG) abordando temas referentes à velhice e ao envelhecimento, aplicado pela própria pesquisadora. Ocorreu no período de 5 dias, com duração de 90 minutos cada encontro, em julho de 2012.

Os temas que compuseram o PEG foram definidos junto à diretoria de saúde do município, aqueles que a pesquisadora julgou importantes e os que faziam parte do questionário de avaliação de conhecimentos gerontológicos. Cinco temas centrais compuseram o PEG: no primeiro encontro foi tratado o tema "envelhecimento humano: possibilidades e desafios", no qual buscou familiarizar os ACS com as nomenclaturas senilidade e senescência, explanações acerca dos aspectos do envelhecimento, demografia e epidemiologia populacional brasileira; no segundo focou-se nas transformações e declínios nos sistemas cardiovascular, respiratório e musculoesquelético; no terceiro foi desenvolvido o tema doença de Alzheimer, com descrição biológica e fases de evolução da doença, sintomas, tratamentos, prevenções; no quarto foi elucidado o assunto sobre relações familiares e sociais dos idosos versos violência contra o idoso e no quinto, e último encontro, foi explanado o tema finitude, destacando aspectos éticos e reflexivos. Todos os encontros desenvolveram-se por meio de aulas expositivas e dialogadas, com vídeos como atividades complementares e estudos e discussão de casos, de idosos que compunham o cotidiano de trabalho dos ACS.

Previamente, foi feito convite aos agentes comunitários para participação ao programa e instruções para preenchimento dos questionários sociodemográfico e de avaliação do contato dos ACS com idosos. Os locais e datas para desenvolvimento do PEG foram definidos junto aos responsáveis pelos agentes comunitários de cada unidade de saúde, sendo realizados em períodos distintos, até finalizar as 12 localidades. Destaca-se que para avaliar o impacto do programa de educação em gerontologia foi empregado o questionário para avaliação de conhecimentos em relação à velhice em momentos diferentes, aplicado antes, imediatamente após e depois de três meses ao desenvolvimento do programa. O tempo gasto em cada aplicação foi 30 minutos. Os ACS foram informados das reaplicações do questionário, com antecipação.

Todas as análises foram feitas no programa SAS para Windows, versão 9.2 e foi adotado nível de significância de $5 \%$ para todos os testes ou p-valor correspondente $(p<0,05)$. As variáveis sociodemográficas quantitativas (idade; renda familiar e tempo de trabalho como ACS) foram analisadas com base nas médias e desvio-padrão. As demais foram categorizadas com base nas frequências e percentuais, encontradas para: estado civil (casados, solteiros, divorciados ou viúvos); nível de escolaridade (ensino fundamental completo, ensino médio incompleto, ensino médio completo, ensino superior incompleto, ensino superior completo) e participação em educação gerontológica (sim ou não).

No questionário de avaliação do contato dos ACS com idosos as variáveis foram categorizadas segundo frequências e percentuais, para: idoso na família, mora/morou com idoso e experiência de trabalho com idosos (sim ou não); frequência de contato com idosos (sempre, às vezes ou nunca); características do contato com idosos (formal, informal ou mista); aquisição da experiência de trabalho com idosos (cotidiano de trabalho como ACS, capacitação do SUS e cotidiano de trabalho ou por meio de cursos/congressos, palestras procuradas por interesse próprio) e contribuição do ACS ao idoso (muito, um pouco ou nada).

No questionário de conhecimentos gerontológicos, cada questão foi avaliada nos momentos ( 1 e 2, 2 e 3,1 e 3) com uso do teste qui-quadrado de tendência. O escore final foi avaliado segundo delineamento 
em medidas repetidas por meio da análise da variância seguida de teste de comparação múltipla de Tukey ajustado ao delineamento. Os dados foram representados em média, desvio-padrão, máximo e mínimo.

O projeto foi aprovado pelo Comitê de Ética em Pesquisa da Faculdade de Medicina de Botucatu (FMB-UNESP), sob o protocolo CEP 4149-2012.

\section{Resultados}

Entre os agentes comunitários predominaram mulheres, casadas, adultos jovens, com ensino médio completo, tempo de trabalho como ACS de 31 meses e com participação em educação gerontológica (Tabela 1).

$\mathrm{Na}$ análise do contato dos ACS com idosos predominou que possuem pelo menos um membro idoso na família $(87,2 \%)$, mora/morou com idosos (62,7\%), sempre possuem contato com esses indivíduos $(95,3 \%)$, sendo a característica desse contato mista $(77,9 \%)$. A maioria dos agentes afirmou ter experiência de trabalho com idosos (93\%), experiência obtida por meio do cotidiano de trabalho $(48,1 \%)$ e acreditam que contribuem muito para promoção da qualidade de vida de idosos (69\%). Os resultados obtidos mediante as avaliações sobre conhecimento gerontológico aos agentes foram relativamente maiores após o desenvolvimento do Programa de Educação em Gerontologia.

Na primeira aplicação do questionário para avaliação de conhecimentos em relação à velhice (antes do desenvolvimento do PEG) houve média de acertos de 10,2 e desvio-padrão de 2,2. Na segunda aplicação do mesmo, desenvolvida imediatamente após o PEG, obteve-se média de 21,1 e desvio-padrão de 1,9 e, na terceira aplicação, ocorrida três meses após o programa, alcançou-se média de 16,4 e desvio-padrão de 3,0.

Tabela 1. Perfil sociodemográfico dos Agentes Comunitários de Saúde, Botucatu, SP, 2013.

\begin{tabular}{|c|c|c|c|c|}
\hline Variáveis & $\mathbf{n}$ & $\%$ & Média & Desvio-padrão \\
\hline Idade (em anos) & & & 34,4 & 8,73 \\
\hline${ }^{*}$ Renda & & & 3 & 1 \\
\hline Tempo de trabalho com ACS (em meses) & & & 31,5 & 22,6 \\
\hline \multicolumn{5}{|l|}{ Sexo } \\
\hline Feminino & 78 & 90,7 & & \\
\hline Masculino & 8 & 9,3 & & \\
\hline \multicolumn{5}{|l|}{ Estado Civil } \\
\hline Casados & 46 & 53,4 & & \\
\hline Solteiros & 25 & 29,0 & & \\
\hline Divorciados & 13 & 15,1 & & \\
\hline Viúvos & 2 & 2,3 & & \\
\hline \multicolumn{5}{|l|}{ Nível de Escolaridade } \\
\hline Ensino Fundamental Completo & 4 & 4,6 & & \\
\hline Ensino Médio Incompleto & 2 & 2,3 & & \\
\hline Ensino Médio Completo & 68 & 79,0 & & \\
\hline Ensino Superior Incompleto & 6 & 6,9 & & \\
\hline Ensino Superior Completo & 3 & 3,4 & & \\
\hline \multicolumn{5}{|l|}{ Participação em Educação Gerontológica } \\
\hline Sim & 53 & 61,6 & & \\
\hline Não & 33 & 38,3 & & \\
\hline
\end{tabular}


O percentual de acertos nas diferentes aplicações do questionário, para cada questão, também foi significativamente maior após o desenvolvimento do PEG. Destaca-se que os resultados obtidos na terceira aplicação foram maiores se comparado à primeira, exceto para a questão de número 16. Reforça-se a importância de educação continuada em gerontologia para agentes comunitários (Gráfico 1).

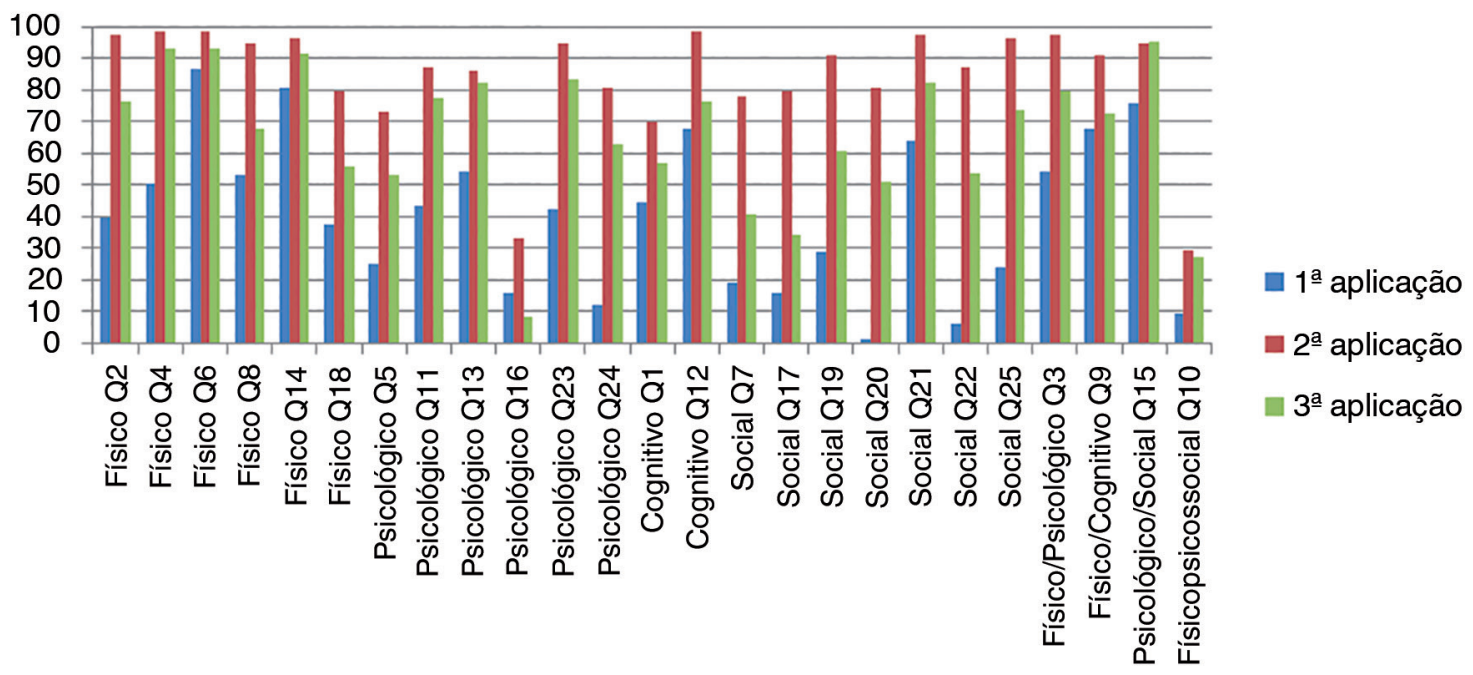

Gráfico 1. Distribuição da frequência relativa de acertos nos diferentes momentos de aplicação do questionário para avaliação de conhecimentos em gerontologia, por questão e domínios, Botucatu, SP, 2013.

Os resultados obtidos mediante a análise de tendência de acertos ao questionário de avaliação de conhecimentos gerontológicos demonstraram maior número de respostas corretas nas aplicações posteriores ao desenvolvimento do PEG, confirmando-se pelo teste de qui-quadrado de tendência $(p<0,05)$. Comparando-se a primeira com a segunda aplicação do questionário, é nítido o aumento de respostas corretas. Na análise da segunda com a terceira aplicação há um decréscimo na quantidade de acertos, entretanto, foi verificado o maior nível de acertos se correlacionada a terceira com a primeira aplicação do mesmo. (Tabela 2).

Antes do desenvolvimento do PEG, os agentes comunitários demonstraram maior percentual de acertos em questões referentes ao domínio físico e, após o programa, obteve-se alto nível de acertos ao questionário relacionado a todos os domínios, sendo estes: físico, cognitivo, psicológico, social e os domínios compostos, físico/psicológico, psicológico/social e físico/psicológico/social.

\section{Discussão}

Apesar da amostra estudada não ser representativa da população de ACS de todo o território brasileiro, os dados obtidos acerca desses profissionais quanto ao perfil sociodemográfico e contato com grupo de idosos estão em consonância com aqueles descritos na literatura e provavelmente refletem a situação geral. O perfil dos agentes comunitários foi predominantemente de mulheres, casados, adultos jovens, com escolaridade média completa, como observado em outros estudos desenvolvidos. ${ }^{10,11}$ Quanto a renda familiar e tempo médio de trabalho como ACS, respectivamente, obteve-se três salários mínimos e aproximadamente dois anos e meio de trabalho. Esses dados também foram verificados em outras pesquisas. ${ }^{10,11}$ 
Tabela 2. Resultado da análise de tendências das diferentes aplicações do questionário para avaliação de conhecimentos em gerontologia, Botucatu, SP, 2013.

\begin{tabular}{|c|c|c|c|c|c|c|c|c|c|c|}
\hline \multirow{2}{*}{ Questões } & \multirow{2}{*}{ Domínios } & \multicolumn{2}{|c|}{ Aplicação 1} & \multicolumn{2}{|c|}{ Aplicação 2} & \multicolumn{2}{|c|}{ Aplicação 3} & \multirow{2}{*}{$\mathbf{p}^{1}$} & \multirow{2}{*}{$\mathbf{p}^{2}$} & \multirow{2}{*}{$\mathbf{p}^{3}$} \\
\hline & & $\mathbf{N}$ & $\%$ & $\mathbf{N}$ & $\%$ & $\mathbf{N}$ & $\%$ & & & \\
\hline Q1 & Cognitivo & 37 & 44,58 & 54 & 70,15 & 48 & 57,14 & $<0,0001^{*}$ & $0,0150^{*}$ & $0,0445^{\star}$ \\
\hline Q2 & Físico & 33 & 39,76 & 76 & 97,44 & 64 & 76,19 & $<0,0001^{*}$ & $<0,0001^{*}$ & $<0,0001^{*}$ \\
\hline Q3 & Físico/psicológico & 45 & 54,22 & 76 & 97,44 & 67 & 79,76 & $<0,0001^{*}$ & $0,0005^{\star}$ & $0,0042^{*}$ \\
\hline Q4 & Físico & 42 & 50,60 & 77 & 98,72 & 78 & 92,86 & $<0,0001^{*}$ & 0,0816 & $<0,0001^{*}$ \\
\hline Q5 & Psicológico & 21 & 25,30 & 57 & 73,08 & 44 & 53,01 & $<0,0001^{*}$ & $0,0251^{*}$ & $0,0010^{\star}$ \\
\hline Q6 & Físico & 72 & 86,75 & 77 & 98,72 & 78 & 92,86 & $<0,0129^{*}$ & 0,0999 & 0,3988 \\
\hline Q7 & Social & 16 & 19,28 & 61 & 78,21 & 34 & 40,48 & $<0,0001^{*}$ & $<0,0001^{*}$ & $0,0002^{*}$ \\
\hline Q8 & Físico & 44 & 53,01 & 74 & 94,87 & 57 & 67,86 & 0,5640 & $0,0009^{*}$ & $0,0246^{*}$ \\
\hline Q9 & Físico/Cognitivo & 56 & 67,47 & 71 & 91,03 & 61 & 72,62 & $<0,0001^{*}$ & $0,0045^{\star}$ & 0,0694 \\
\hline Q10 & Psicológico/social/Físico & 8 & 9,64 & 23 & 29,49 & 23 & 27,38 & 0,1458 & 0,1420 & 0,7874 \\
\hline Q11 & Psicológico & 36 & 43,37 & 67 & 87,01 & 65 & 77,38 & $<0,0001^{*}$ & 0,0750 & $0,0290^{\star}$ \\
\hline Q12 & Cognitivo & 56 & 67,47 & 77 & 98,72 & 64 & 76,19 & $<0,0001^{*}$ & $<0,0001^{*}$ & 0,4007 \\
\hline Q13 & Psicológico & 45 & 54,22 & 67 & 85,90 & 69 & 82,14 & $<0,0001^{*}$ & 0,5438 & $0,0004^{*}$ \\
\hline Q14 & Físico & 67 & 80,72 & 75 & 96,15 & 77 & 91,67 & $0,0064^{*}$ & 0,1892 & 0,0920 \\
\hline Q15 & Psicológico/Social & 63 & 75,90 & 74 & 94,87 & 80 & 95,24 & $0,0009^{\star}$ & 0,6558 & $0,0014^{*}$ \\
\hline Q16 & Psicológico & 13 & 15,66 & 26 & 33,33 & 7 & 8,33 & $0,0138^{*}$ & $<0,0001^{*}$ & 0,0785 \\
\hline Q17 & Social & 13 & 15,66 & 62 & 79,49 & 29 & 34,52 & $<0,0001^{*}$ & $<0,0001^{*}$ & 0,2797 \\
\hline Q18 & Físico & 31 & 37,35 & 62 & 79,49 & 47 & 55,95 & $<0,0001^{*}$ & $0,0017^{*}$ & $0,0404^{*}$ \\
\hline Q19 & Social & 24 & 28,92 & 70 & 90,91 & 51 & 60,71 & 0,0658 & 0,2027 & 0,4583 \\
\hline Q20 & Social & 1 & 1,20 & 63 & 80,77 & 43 & 51,19 & $<0,0001^{*}$ & $0,0010^{*}$ & $<0,0001^{*}$ \\
\hline Q21 & Social & 53 & 63,86 & 76 & 97,44 & 69 & 82,14 & $<0,0001^{*}$ & $0,0015^{\star}$ & $0,0077^{*}$ \\
\hline Q22 & Social & 5 & 6,02 & 68 & 87,18 & 45 & 53,57 & $<0,0001^{*}$ & 0,9237 & $<0,0001^{*}$ \\
\hline Q23 & Psicológico & 35 & 42,17 & 74 & 94,87 & 69 & 83,13 & $<0,0001^{*}$ & $0,0318^{*}$ & $<0,0001^{*}$ \\
\hline Q24 & Psicológico & 10 & 12,20 & 63 & 80,77 & 53 & 63,10 & $<0,0001^{\star}$ & $0,0002^{*}$ & $<0,0001^{*}$ \\
\hline Q25 & Social & 20 & 24,10 & 75 & 96,15 & 62 & 73,81 & $<0,0001^{*}$ & $0,0004^{\star}$ & $<0,0001^{*}$ \\
\hline
\end{tabular}

* Nível descritivo significante em $a \leq 0,05$. Legenda: $\mathrm{P}^{1}$ : valor $\mathrm{p}$ referente à tendência de acertos da $1^{\text {a }}$ aplicação para $2^{\underline{a}}$ aplicação; $\mathrm{P}^{2}$ : valor $\mathrm{p}$ referente à

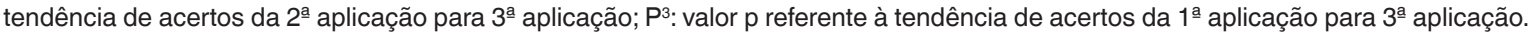

De acordo com dados sobre contato dos ACS com idosos, foi observado que a maioria deles possui um membro idoso na família e já morou ou ainda mora com alguma pessoa idosa. Também expuseram sempre ter contato com idosos, sendo este contato caracterizado como misto, ou seja, estabelecido na rotina de trabalho, bem como no âmbito familiar. Esses dados estão em consonância com os obtidos em estudo realizado por Ferreira \& Ruiz, em que 53\% dos ACS entrevistados moravam ou moraram com idosos. ${ }^{6}$ Nessa temática, a maioria dos agentes acredita possuir experiência para trabalhar com idosos, disseram ter adquirido experiência no cotidiano de trabalho e por educações gerontológicas oferecidas pelo SUS. Mais da metade deles afirmou já ter participado de alguma capacitação envolvendo o tema sobre idosos. Em outro estudo foi verificado que aproximadamente $45 \%$ dos agentes tiveram capacitação em saúde para idosos, contudo, foi observado maior índice de participações em capacitações envolvendo o tema idoso entre aqueles agentes que possuíam maior tempo de trabalho na função. ${ }^{6}$

A propósito de educação gerontológica destinada aos ACS, alguns dos dados obtidos neste estudo remetem à seguinte reflexão: embora a maioria dos agentes afirmasse ter participado de educação em 
gerontologia, metade deles alegou ter adquirido experiência na atenção aos idosos, somente por meio do cotidiano de trabalho. Isto se torna preocupante, na medida em que a população de idosos aumenta exponencialmente na sociedade brasileira e os ACS não estejam ainda preparados para atender essa demanda. Segundo Fernandes et al., ${ }^{12}$ em estudo realizado em município paulista, $88 \%$ dos ACS da amostra não havia realizado curso na área de gerontologia, apesar de eles referirem bastante contato com idosos. Nesse estudo foi observado que os ACS demonstraram maior conhecimento prévio acerca do domínio físico, diagnosticado pela aplicação do questionário para avaliação de conhecimentos gerontológicos realizada no início do estudo.

Segundo Ferreira \& Ruiz, ${ }^{6}$ o maior conhecimento de temas relacionados aos aspectos físicos do envelhecimento e da velhice, em detrimento de aspectos psicológicos, cognitivos e sociais, mostra que, apesar dos ACS estarem inseridos no contexto de vida dos usuários idosos nas unidades de saúde, é preponderante o conhecimento biológico sobre os idosos. Conforme Nunes et al., ${ }^{13}$ os agentes comunitários, por fazerem parte da equipe de saúde, são levados a se identificar com certos conteúdos e práticas referentes à biomedicina. Pelo convívio com profissionais da saúde, os agentes acabam tendo maior acesso ao saber biomédico e o valorizando mais que o conhecimento popular. A busca de formação técnica confirma essa visão ilusória que com conhecimentos biomédicos estariam mais capacitados para resolver os problemas da comunidade.

Nesse sentido, a educação continuada e ou permanente para agentes comunitários deve muni-los de conhecimentos diversos em torno da questão do processo de saúde-doença, incorporando, além da perspectiva biomédica, outros saberes que o habilitem na interação com as famílias e no reconhecimento de suas necessidades. ${ }^{13}$ A preocupação quanto à educação continuada em saúde destinada ao ACS é manifestada pelo próprio agente, como evidenciada no estudo realizado por Marzari et al., ${ }^{14}$ em que um dos entrevistados relatou que "o que nos falta é embasamento teórico para informar a população". Imediatamente após o desenvolvimento do programa de educação em gerontologia foi reaplicado o instrumento para avaliação de conhecimentos gerontológicos, sendo nesse momento verificado o alto índice de acertos em praticamente todas as questões que o compunham. Apesar de este resultado ser esperado, todavia possibilita a reflexão da importância de educação continuada com essa finalidade, aos agentes comunitários.

Segundo Ferreira \& Ruiz, ${ }^{6}$ a capacitação prévia em envelhecimento, para ACS, esteve relacionada aos melhores resultados obtido com aplicação do mesmo questionário. E ainda conforme Neri \& Jorge, ${ }^{15}$ em estudo realizado com alunos de educação física, pedagogia, enfermagem e medicina, mostrou que a aproximação teórica dos estudantes com temas sobre envelhecimento também produziu maior acerto ao questionário. No presente estudo, a falta de continuidade no desenvolvimento do programa de educação em gerontologia, que constou do total de cinco encontros, foi um dos determinantes para diminuição no nível de acertos ao questionário, entretanto, outros motivos poderiam estar associados como, por exemplo, ao baixo nível de escolaridade representado por 4,6\% dos ACS. Tal fato reflete um limitante do estudo, como também a inaplicação do instrumento de avaliação em conhecimentos gerontológicos em outros períodos, bem como a impossibilidade de análise do impacto da educação gerontólogica na assistência prestada pelos agentes aos idosos. 
Entretanto, verificou-se que na terceira aplicação do questionário, ocorrida três meses após o desenvolvimento da educação gerontológica, obtiveram-se níveis de acerto maior, em todos os domínios, se comparada à primeira aplicação do mesmo, que ocorreu antes do programa. Exceto para a questão de número 16, que tratava do tema sobre emoção comparando-se jovens e idosos, enquadrada no domínio psicológico. Mediante esse fato, em estudo realizado por Cachioni, ${ }^{16} \mathrm{com}$ aplicação do mesmo questionário em sua amostra, a autora pôde constatar menor nível de acertos demonstrado em questões de domínio psicológico e social. No presente estudo tal fato talvez possa ser explicado pela insistência dos agentes em assinalar a resposta que melhor condizia às experiências de trabalho com idosos.

Essa perspectiva, a Política Nacional de Educação Permanente em Saúde (Pneps) enfoca a educação no serviço como o tipo de formação "mais apropriado para produzir as transformações nas práticas e nos contextos de trabalho, fortalecendo a reflexão na ação, o trabalho em equipe e a capacidade de gestão sobre os próprios processos locais". ${ }^{17}$ Conforme Bachilli, ${ }^{18}$ a figura do agente comunitário de saúde surge das comunidades no contexto da atenção primária em saúde no Brasil, sem qualquer bagagem técnica para o pleno exercício de suas atividades, assumindo a importante função de interlocução entre equipe de saúde e comunidade local.

Assim, enfocar as competências na atuação junto ao idoso objetiva novos recortes do conhecimento e sua contextualização no processo social do envelhecimento e na prestação de serviços. Inclui a capacidade de atuação frente à imprevisibilidade e diversidade de situações, almeja o trabalho em equipe interdisciplinar e mobilização de conteúdos diversos buscando atuação integral ao nível do profissional de saúde, das estruturas organizacionais, dos arranjos políticos. ${ }^{19}$ Desta maneira, ressalta-se a necessidade de estudos futuros com enfoque nos agentes comunitários na perspectiva de promoção de educação continuada e/ou permanente em temas gerontológicos, visando à atenção ao idoso com qualidade.

\section{Conclusão}

Oferecer educação continuada em gerontologia, ou seja, temas específicos sobre envelhecimento e velhice, para agentes comunitários de saúde é necessário e funciona, visto que há lacunas em conhecimento gerontológico por esses profissionais. Uma vez providos de conhecimentos, os agentes podem reforçar seu papel mediante a equipe de saúde e população, dentre ela os idosos.

\section{Referências}

1. Lebrão ML, Duarte YAO. SABE- Saúde, Bem-Estar e envelhecimento - o projeto SABE no município de São Paulo: uma abordagem inicial. Brasília: Organização Pan-Americana de Saúde, 2003, p. 75-91.

2. Schmidt MI, Duncan BB, Azevedo e Silva G, Menezes AM, Monteiro CA, Barreto SM, et al. Chronic non-communicable diseases in Brazil: burden and current challenges. Lancet. 2011;377(9781):1949-61. http://dx.doi.org/10.1016/S01406736(11)60433-9

3. Vecchia MD. A saúde mental no Programa da Saúde da Família: estudo sobre práticas e significações de uma equipe (Dissertação de mestrado). Botucatu: Faculdade de Medicina de Botucatu, Universidade Estadual São Paulo, Júlio de Mesquita Filho, Botucatu; 2006.

4. Brasil. Ministério da Saúde. Guia Prático do Programa Saúde da Família. Brasília: Ministério da Saúde; 2001.69p [Acesso 13 Ago 2015]. Disponível em: http://dab.saude.gov.br/portaldab/biblioteca.php?conteudo=publicacoes/guia_pratico_ saude_familia 
5. Bezerra AFB, Espírito Santo ACG, Batista Filho M. Concepções e práticas do agente comunitário de saúde na atenção à saúde do idoso. Rev Saúde Pública. 2005;39(5):809-15. DOI: http://dx.doi.org/10.1590/S0034-89102005000500017

6. Ferreira VM, Ruiz T. Atitudes e conhecimentos de agentes comunitários de saúde e suas relações com idosos. Rev Saúde Pública. 2012;46(5):849-3. DOI: http://dx.doi.org/10.1590/S0034-89102012000500011

7. Peduzzi M, Guerra DAD, Braga CP, Lucena FS, Silva, JAM. Atividades educativas de trabalhadores na atenção primária: concepções de educação permanente e de educação continuada em saúde presentes no cotidiano de unidades básicas de saúde em São Paulo. Interface Comun Saúde Educ. 2009;13(30):121-34. DOI: http://dx.doi.org/10.1590/S141432832009000300011

8. Instituto Brasileiro de Geografia e Estatística (IBGE). Censo demográfico 2010: características gerais da população, religião e pessoas com deficiência. Rio de Janeiro, 2010.125p [Acesso 13 Ago 2015]. Disponível em: http://www.ibge.gov.br/home/ estatistica/populacao/censo2010/caracteristicas_religiao_deficiencia/default_caracteristicas_religiao_deficiencia.shtm

9. Harris DK, Changas PS, Palmore EB. Palmore's first facts on aging quiz in a multiple-choice format. Educ Gerontol. 1996;22(6):575-89. DOI: http://dx. doi.org/10.1080/0360127960220605

10. Ferraz L, Aertz DRGC. O cotidiano do agente comunitário de saúde no PSF em Porto Alegre. Rev Ciência Saúde Coletiva. 2005;10(2):347-55. DOI: http://dx.doi.org/10.1590/S1413-81232005000200012

11. Ursine BL, Trella CS, Nunes EFPA. O agente comunitário de saúde na estratégia saúde da família: uma investigação das condições de trabalho e da qualidade de vida. Rev Bras Saúde Ocup. 2010;35(122):339-27. DOI: http://dx.doi.org/10.1590/ S0303-76572010000200015

12. Fernandes HCL, Pavarini SCI, Barham EJ, Mendiondo MSZ, Luchesi BM. Envelhecimento e demência: o que sabem os agentes comunitários de saúde? Rev Esc Enferm USP. 2010;44(3):782-8. DOI: http://dx.doi.org/10.1590/S008062342010000300033

13. Nunes MO, Trad LB, Almeida BA, Homem CR, Melo MCIC. O agente comunitário de saúde: construção da identidade desse personagem híbrido e polifônico. Cad Saúde Pública. 2002;18(6):1646-39. DOI: http://dx.doi.org/10.1590/S0102$311 \times 2002000600018$

14. Marzari CK, Junges JR, Selli L. Agentes comunitários de saúde: perfil e formação. Rev Ciên Saúde Coletiva. 2011;16(1):88073. DOI: http://dx.doi.org/10.1590/S1413-81232011000700019

15. Neri AL, Jorge MD. Atitudes e conhecimentos em relação à velhice em estudantes de graduação em educação e em saúde: subsídios ao planejamento curricular. Estud Psicol. 2006;23(2):127-37. DOI: http://dx.doi.org/10.1590/S0103166X2006000200003

16. Cachioni M. Quem educa os idosos?: um estudo sobre professores de Universidades da terceira idade. Campinas: Alínea; 2003. p.155-75.

17. Pedrosa IL, Lira GA, Oliveira B, Silva MS, Santos MB, Silva EA, et al. Uso de metodologias ativas na formação técnica do agente comunitário de saúde. Trab Educ Saúde. 2011;9(2):319-32. DOI: http://dx.doi.org/10.1590/S198177462011000200009

18. Bachilli RG, Scavassa AJ, Spiri WC. A identidade do agente comunitário de saúde: uma abordagem fenomenológica. Ciênc Saúde Coletiva. 2008;13(1):51-60. DOI: http://dx.doi.org/10.1590/S1413-81232008000100010

19. Motta LB, Aguiar AC. Novas competências profissionais em saúde e o envelhecimento populacional brasileiro: integralidade, interdisciplinaridade e intersetorialidade. Ciênc Saúde Coletiva. 2007;12(2):363-72. DOI: http://dx.doi.org/10.1590/S141381232007000200012 